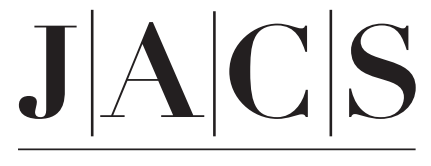

A R T I C L E S

Published on Web 08/31/2005

\title{
Supercapacitive Admittance Tomoscopy
}

\author{
Jean Gamby, Jean-Pierre Abid, and Hubert H. Girault* \\ Laboratoire d'Electrochimie Physique et Analytique, Ecole Polytechnique Fédérale de Lausanne, \\ CH 1015- Lausanne, Switzerland \\ Received January 29, 2005; E-mail: hubert.girault@epfl.ch
}

\begin{abstract}
A sensor for measuring adsorption on a substrate has been designed including a contactless detection scheme, called supercapacitive admittance tomoscopy (SCAT). The sensor comprises a thin dielectric layer with two parallel band electrodes on the one side and a chemically modified surface on the other onto which the adsorption of molecules occurs. Upon application of a high frequency ac voltage between the two electrodes, a capacitive coupling is established across the dielectric layer, and the admittance measured depends on the surface state of the chemically modified interface. On the basis of this principle, a flow sensor has been developed to measure sensorgrams to follow the dynamics of the adsorption and has been tested for the adsorption of IgG on the modified surface.
\end{abstract}

\section{Introduction}

The measurements of noncovalent interactions address a fundamental aspect of life science, and different methods have been developed to study biomolecular interactions, such as protein-protein interactions. To measure these interactions, one possible route involves immobilizing one of the two species on chemically modified surfaces, sometimes called the biosensor route, by opposition to methods measuring these interactions in the bulk ${ }^{1}$. The detection tools used in this type of biosensor design include both electrochemical ${ }^{2-5}$ and/or spectroscopic principles. Among them, optical techniques such as confocal fluorescence microscopy, ${ }^{6}$ surface plasmon resonance, ${ }^{7,8}$ and electrochemical techniques ${ }^{11,12}$ have been proposed for studying the adsorption of target molecules onto solid surfaces depending on the nature of the surface. The most widely used technique is based on surface plasmon resonance (SPR) measurements and is routinely used for monitoring the adsorption of target biomolecules on specifically prepared glass slides containing a thin gold film. ${ }^{13}$ Recently, a new combined technique called surface plasmon resonance/Love mode surface acoustic wave

(1) Kluger, R.; Zhang, J. J. Am. Chem. Soc. 2003, 125 (20), 6070-6071.

(2) Katz, E.; Bückmann, A. F.; Willner, I. J. Am. Chem. Soc. 2001, 123 (43), 10752-10753.

(3) Harrell, C. C.; Kohli, P.; Siwy, Z.; Martin, C. R. J. Am. Chem. Soc. 2004, $126,15646-15647$.

(4) Huang, Y.; Duan, X.; Cui, Y.; Lauhn, L. J.; Kim, K. H.; Lieber, C. M. Science 2001, 294, 1313-1317.

(5) Cui, Y.; Wei, Q.; Park, H.; Lieber, C. M. Science 2001, 293, 1289-1292.

(6) O'Brien, J. C.; Jones, V. W.; Porter, M. D. Anal. Chem. 2000, 72, 703710.

(7) Wegner, G. J.; Wark, A. W.; Lee, H. J.; Codner, E.; Saeki, T.; Fang, S.; Corn, R. M. Anal. Chem. 2004, 76, 5677-5684.

(8) Mullett, W. M.; Lai, E. P. C.; Yeung, J. M. Methods 2000, 22, 77-91.

(9) Ditcham, W. G. F.; Al-Obaidi, A. H. R.; McStay, D.; Mottram, T. T.; Brownlie, J.; Thompson, I. Biosens. Bioelectron. 2001, 16, 221-224.

(10) McStay, D.; Yang, L. C.; Quinn, P. J. Sensors and their Applications; Institute of Physics: Bristol, U.K., 1995; Vol. 7.

(11) Sargent, A.; Sadik, O. A. Anal. Chim. Acta 1998, 376, 125-131.

(12) Doyl, J. M.; Wehmeyer, K. R.; Heineman, W. R.; Halsall, H. B. Electrochemical Sensors in Immunological Analysis; Plenum Press: New York, 1987; p 87.

(13) Yonson, C. R.; Jeoung, E.; Zou, S.; Schatz, G. C.; Mrksich, M.; Van Duyne, R. P. J. Am. Chem. Soc. 2004, 126 (39), 12669-76.

13300 — J. AM. CHEM. SOC. 2005, 127, 13300-13304 has emerged for investigating the adsorption of biomolecules at the solid/liquid interface. ${ }^{14}$ In comparison with the SPR technique, a modification is made on the quartz substrate, which is patterned with a double-finger interdigitated electrode for launching a Love mode acoustic wave at a frequency of 123.5 MHz. During the process of adsorption, the modification of the phase shift can be converted to a frequency shift and, thus, related to the bound mass of biomolecules. A similar approach can be envisaged using a quartz balance for monitoring the adsorption process of biomolecules and, thus, estimating the quantity of molecules adsorbed on the substrate. The principle is based on following any variation of the resonance frequency due to the protein adsorption at the surface of the quartz crystal, which gives an indication of the quantity of molecules adsorbed on the sensor surface. ${ }^{15}$

Electrochemical methods such as impedance spectroscopy have also been investigated for probing biomolecular interactions at conductive and semiconductive surfaces with related applications, such as immunosensors and DNA and enzyme biosensors. ${ }^{16,17}$ In that configuration, the bias of the electrode potential is controlled, and the frequency of the alternating potential difference imposed is in the kilohertz range.

In the field of separation science, e.g., chromatography or electrophoresis, conductometric techniques are widely used for the detection of ions. ${ }^{18}$ Noncontact systems, which used to be called oscillometry, were reintroduced more recently for the detection of low concentrations of ions in capillary electrophoresis. ${ }^{19 a-d}$ Similarly, noncontact impedance measurement

(14) Zhou, C.; Friedt, J. M.; Angelova, A.; Choi, K. H.; Laureyn, W.; Frederix, F.; Francis, L. A.; Campitelli, A.; Engelborghs, Y.; Borghs, G. Langmuir 2004, 20, 5870-5878.

(15) (a) Höök, F.; Rodahl, M.; Brzezinski, P.; Kasemo, B. Langmuir 1998, 14, 729-734. (b) Rodahl, M.; Kasemo, B. Sens. Actuators, A 1998, 54, 448456.

(16) Tanious, F. A.; Hamelberg, D.; Bailly, C.; Czarny, A.; Boykin, D. W.; Wilson, W. D. J. Am. Chem. Soc. 2004, 126 (1), 143-53.

(17) Hickman, J. J.; Ofer, D.; Laibinis, P. E.; Whitesides, G. M.; Wrighton, M. S. Science 1991, 252, 688.

(18) De Vries, G. Nature 1954, 173, 735-736. 
techniques have been developed as a medical-imaging tool. This group of techniques, referred to as electrical tomography, has been proposed for in vivo imaging processes such as brain activity, ${ }^{20}$ and the absence of risk for the human body opens new applications in several areas of medicine.

The aim of this work is to present a sensitive noncontact technique for monitoring the adsorption of molecules on a chemically modified substrate. The concept is based on establishing a capacitive coupling between two electrodes placed on one side of a dielectric substrate and the solution in contact with the other side. To ensure that a capacitive coupling occurs, we have used electrodes with a high specific surface area following the principle used to develop supercapacitors for energy storage. ${ }^{21}$ Additionally, the surface of the dielectric layer in contact with the solution of analytes to be studied has been modified physically by techniques such as UV laser photoablation or plasma etching to increase the surface roughness and create surface charges by oxidation. The surface can also be chemically treated by oxidizing agents and/or modified by the adsorption of nanoparticles having ionisable groups on their outer layer.

\section{Materials and Methods}

The present biosensor concept comprises a flow channel microfabricated in a poly(ethylene terephthalate) (PET) film, $100 \mu \mathrm{m}$ thick. The flow microchannel was photoablated on one side of the film with a trapezoidal cross-sectional shape and a depth of $45 \mu \mathrm{m}$, a width of $100 \mu \mathrm{m}$, and a length of $1.4 \mathrm{~cm}$. The microstructures were obtained using the projection beam of an ArF excimer laser system (Lambda Physik LPX 205-i) operating at $193 \mathrm{~nm}$ through a rectangular freestanding molybdenum mask. The geometry of the microchannel is designed using computer controlled $X-Y$ translation stages (Physik Instrumente, Germany).

On the other side of the film, two parallel microchannels of the same dimension as the flow channel were photoablated perpendicularly to it as portrayed in Figure 1. The distance separation between these two parallel microchannels is $200 \mu \mathrm{m}$ center to center. The two channels were then filled with a commercial graphite ink (Electra $\Omega$ ED5000 series obtained from Electra Polymers, England) in which gold nanoparticles (19 $\mathrm{nm}$ diameter) were mixed in order to increase the charging capacity (an aliquot of $50 \mu \mathrm{L}$ of colloidal solution containing $48 \mathrm{mg} \mathrm{L}^{-1}$ of gold nanoparticles is added to $500 \mathrm{mg}$ of graphite ink). After curing at $60^{\circ} \mathrm{C}$ for $4 \mathrm{~h}$, the PET film with a flow channel on one side and two conductive tracks on the other side was thermally laminated on both sides with a polyethylene/poly(ethylene terephthalate) (PE/PET) layer with a thickness of $35 \mu \mathrm{m}$ (Morane, Oxon, U.K.) at $135{ }^{\circ} \mathrm{C}$ and a pressure of $2 \mathrm{bar}$. The separation distance across the film between the two conductive tracks acting as band electrodes and the main flow microchannel is equal to $5 \mu \mathrm{m}$, and the detection surface area per microelectrode as outlined using the black lines in Figure 1c is $66 \times 100 \mu \mathrm{m}^{2}$.

The flow microchannel is coated with gold nanoparticles with an average size of $19 \mathrm{~nm}$ and a standard deviation of $2 \mathrm{~nm}$. The colloidal solutions are prepared by reducing a tetrachloraurate salt with trisodium citrate. $^{22}$ The aqueous solution presents a rubis red color and is then

(19) (a) Fracassi da Silva, J. A.; Guzman, N.; Do Lago, C. L. J. Chromatogr., A 2002, 942, 249-258. (b) Zemann, A. J. Trends Anal. Chem. 2001, 20 (6-7), 346-354. (c) Mayrhofer, K.; Zemann, A. J.; Schnell, E.; Bonn, G. K. Anal. Chem. 1999, 71, 3828-3833. (d) Zemann, A. J.; Schnell, E.; Volgger, D.; Bonn, G. K. Anal. Chem. 1998, 70, 536-567.

(20) Ridderinkhof, K. R.; de Vlugt, Y.; Bramlage, A.; Spaan, M.; Elton, M.; Snel, J.; Band, G. P. H. Science 2002, 298, 2209-2211.

(21) Shukla, A. K.; Sampath, S.; Vijayamohanan, K. Curr. Sci. 2000, 79, 16561661.

(22) Turkevich, J.; Stevenson, P. C.; Hillier, J. Discuss. Faraday Soc. 1951, 11,55 .

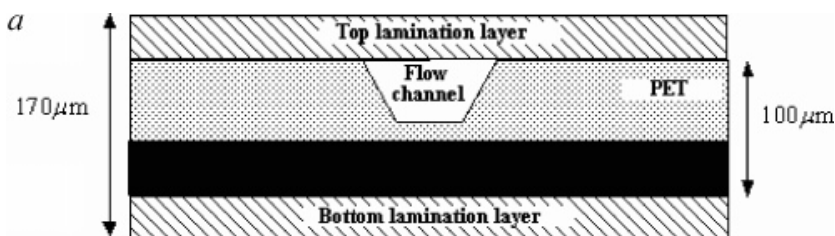

$b$

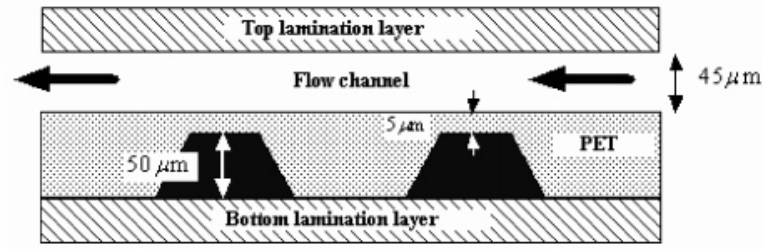

c

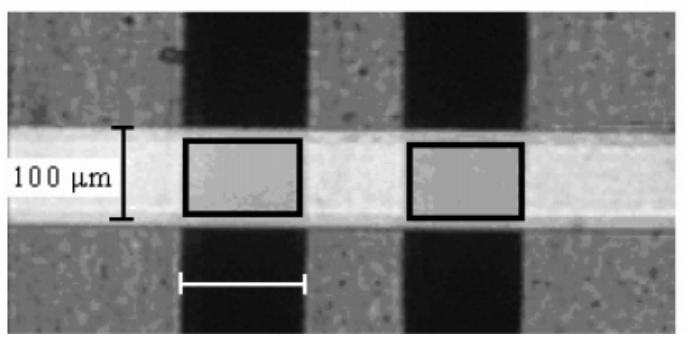

Figure 1. (a) Cut front view of the device across a band electrode. (b) Cross-sectional view of the device; in black, trapezoidal cross sections of the band electrodes. (c) Top view picture obtained by an inverted microscope. Vertically, the two band electrodes beneath the horizontal flow channel are filled with fluorescein sodium $\left(\lambda_{\mathrm{exc}}=490 \mathrm{~nm}, \lambda_{\text {emission }}=514\right.$ $\mathrm{nm})$. The black rectangles represent the area where the capacitive coupling takes place.

treated by cetyl trimethylammonium chloride, yielding positively charged particles, as reported by Weiser. ${ }^{23}$ The coating of the PET channel is achieved by filling the microchannel with a solution of gold nanoparticles and drying it overnight. Finally, to remove unattached nanoparticles, the microchannel is rinsed with MilliQ water. Phosphate buffer saline (PBS) is purchased from Fluka Chemie, Switzerland, and $\mathrm{IgG}$ antirabbit immunoglobulin was provided from Amersham Pharmacia Biotech.

The admittance measurement has been performed by applying between the two band electrodes an ac modulated signal with a frequency ranging from $1 \mathrm{kHz}$ to $1 \mathrm{MHz}$ and an amplitude of $3 \mathrm{~V}$. The current measured is thus related to the total admittance of the sensor. The admittance measurement is performed using a frequency response analyzer (FRA 1255B, Solartron U.K.) together with a dielectric interface 1296 (Solartron, U.K.) which extends the frequency range from $10 \mu \mathrm{Hz}$ to $10 \mathrm{MHz}$. The current measurement range of the system varies from $6 \mathrm{~mA}$ to $100 \mathrm{fA}$, thus enabling the measurements of very high impedance (from $100 \Omega$ to $100 \mathrm{~T} \Omega$ ) and low capacitance (from $1 \mathrm{pF}$ to higher than $0.1 \mathrm{~F}$ ). Data acquisitions are collected using the company-made software SMaRT. In that way, the admittance measurement between the two band electrodes depends on the surface state of the flow channel and the adsorption taking place on the walls of the flow channel.

The principle of supercapacitive coupling across the thin PET layer separating the band electrodes to the flowing solution is to form across this thin layer a capacitor as illustrated in Scheme 1a, where the charges on one side are electronic charges on the band electrodes and the charges on the other side are ionic charges from the ionized groups generated on the PET surface by the photoablation process and more generally by the space-charged region in solution, e.g., the Gouy-Chapman layer. We have shown in the past that microchannels photoablated in PET films have a $\xi$-potential close to that of silica and that microchannels

(23) Weiser, H. B. Inorganic Colloid Chemistry; Wiley: New York, 1933; Vol. 1 , pp $21-57$ 


\section{Scheme 1}

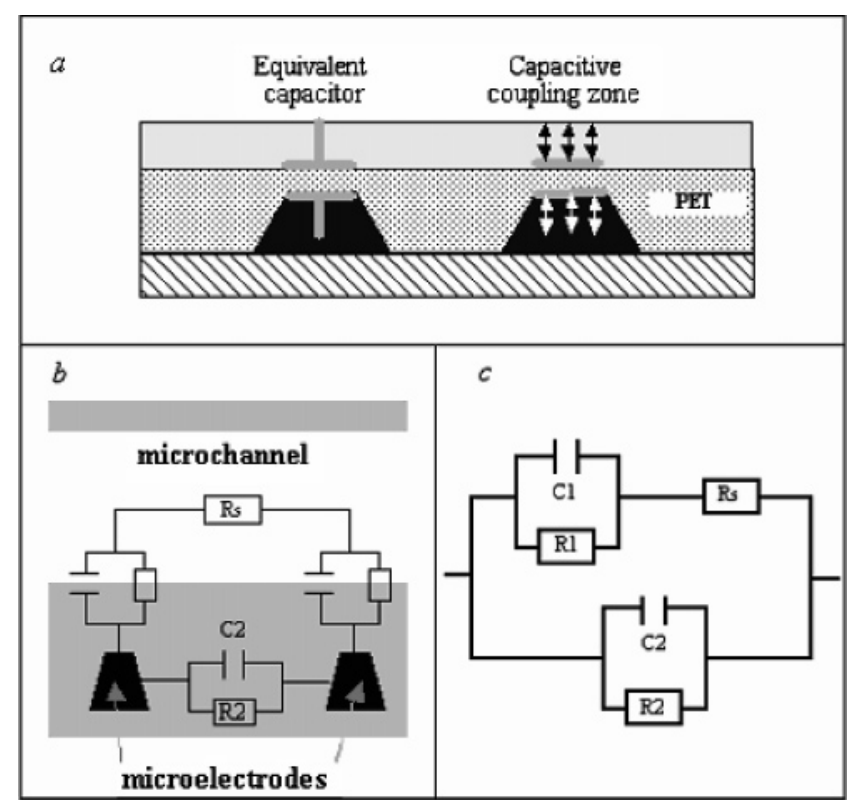

(a) Scheme of the supercapacitive coupling for a contactless device in a high-frequency regime. (b) Schematic representation of the microelectrodes/ PET/microchannels interface. (c) Electrical equivalent circuit of the device.

in PET can be used to induce electro-osmotic pumping and to carry out capillary electrophoresis. ${ }^{24 a, b}$ In comparison to classical impedimetric sensors using a metallic electrode in direct contact with the analyte, we have here a contactless system where the solution is separated from the electrodes by a thin PET layer ( $5 \mu \mathrm{m}$ thick).

For this system to be sensitive, we have to increase the charging ability of the two sides. On the electronic conductor side, we use a graphite ink loaded with gold nanoparticles so as to have a very high specific area. On the aqueous side, the specific surface area is inherently higher than the geometric one due to the photoablation process. Nevertheless, it is possible to improve the sensitivity of the device by adsorbing charged nanoparticles on the walls of the flow channel. Any further chemical modification of the surface charge induces changes of the charge density, which in turn modifies the capacitance of the device. The admittance spectroscopy measurements are therefore relative to a thin layer of solution in the vicinity of the substrate, and this is the reason we have called this detection method "supercapacitive admittance tomoscopy" (tomo refers to the thin layer of solution, and scopy refers to the observation).

\section{Results and Discussion}

Admittance spectroscopy experiments with an ac voltage of $3 \mathrm{~V}$ have been performed for the better understanding of the processes involved in this detection approach. With the use of the configuration described above, admittance measurements are performed on the device filled with a buffer solution, and the real and imaginary parts of the admittance are displayed in Figure 2. As shown in Figure 2b (inset), an increase of the admittance with increasing frequency is observed with a main contribution from the imaginary part.

The evolution of the admittance establishes clearly that the device can be regarded as a capacitive system, where the capacitive coupling takes place at frequencies higher than 10 $\mathrm{kHz}$ and the resistive component is negligible below $500 \mathrm{kHz}$ (see inset of Figure 2). It is important to stress that a capacitive

(24) (a) Bianchi, F.; Chevelot, Y.; Mathieu, H. J.; Girault, H. H. Anal. Chem. 2001, 73, 3845. (b) Roberts, M. A.; Rossier, J. S.; Bercier, P.; Girault, H. H. Anal. Chem. 1997, 69, 2035.

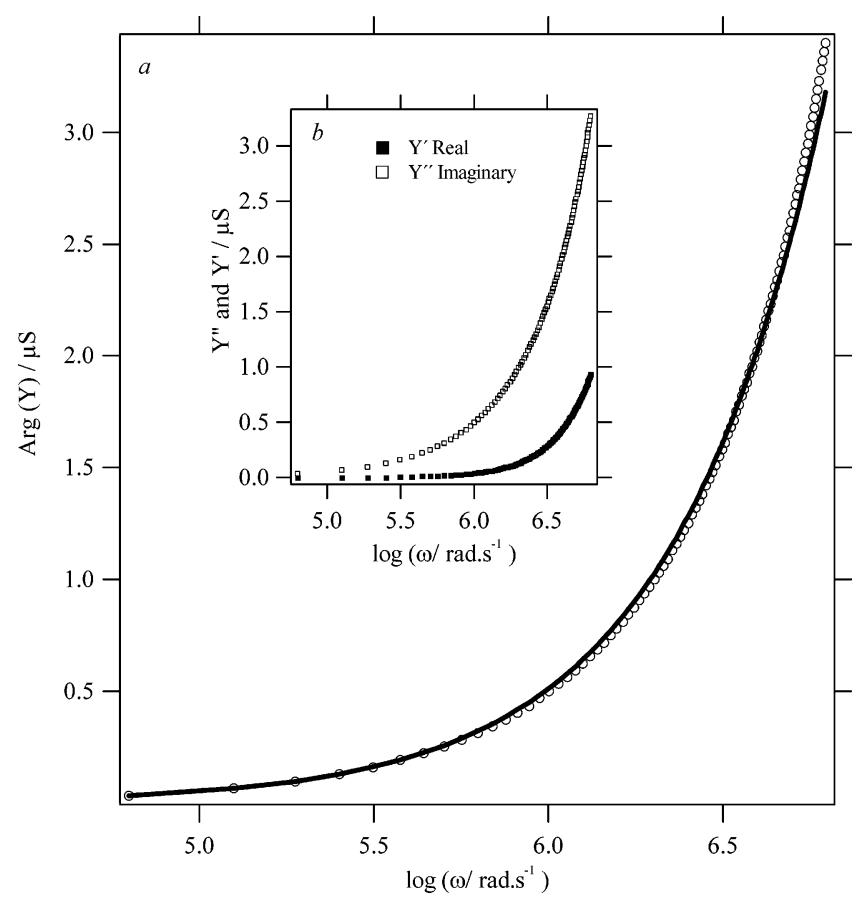

Figure 2. (a) Experimental angular frequency response of the admittance (hollowed spheres) and theoretical fit using eq 1 (solid line). (b) Evolution of the real (filled squares) and imaginary (hollowed squares) parts of the admittance for a buffer-filled channel.

response is the signature of a surface phenomenon and a resistive response is the signature of a bulk response. Indeed, charges can only accumulate at surfaces, bulk being always neutral by definition.

To further explain the variation of the admittance with respect to the frequency, the results are modeled using an equivalent circuit.

From an electrical viewpoint, the equivalent circuit can be considered as a combination of capacitors and resistors. In the present case, due to the presence of dielectric/electrode interfaces, the equivalent circuit shown in Scheme 1 has been envisaged.

For this equivalent circuit, the corresponding admittance is obtained:

$$
Y(\omega)=\frac{1+j \omega R_{2} C_{2}}{R_{2}}+\frac{1+j \omega R_{1} C_{1}}{R_{\mathrm{S}}+R_{1}+j \omega R_{\mathrm{S}} R_{1} C_{1}}
$$

where $R_{1}, R_{2}$, and $R_{\mathrm{S}}$ correspond, respectively, to the resistance of the thin PET dielectric layer between the electrodes and the channel, the interelectrodes resistance through the bulk of the PET substrate, and the solution resistance in the channel. $C_{1}$ is the capacitance of the electrode/PET/channel interface, and $C_{2}$ is the stray capacitance between the electrodes. The best fit for the data of Figure 2 yields the following parameters: $R_{\mathrm{S}}=2220$ $\Omega, R_{1}=2 \times 10^{8} \Omega, R_{2}=2 \times 10^{14} \Omega, C_{1}=5.2 \times 10^{-13} \mathrm{~F}$, and $C_{2}=1 \times 10^{-15} \mathrm{~F}$ (solid line in Figure 2a).

In that configuration, it must be pointed out that the modification of the channel surface results in a modification of the capacitance of the electrode/PET/channel capacitor. With the use of eq 1 , the simulated admittance evolution for different values of $C_{1}$ is portrayed in Figure 3. When the electrode/PET/ channel capacitance is increased, an enhancement of the admittance is observed for frequencies higher than $10 \mathrm{kHz}$, as 


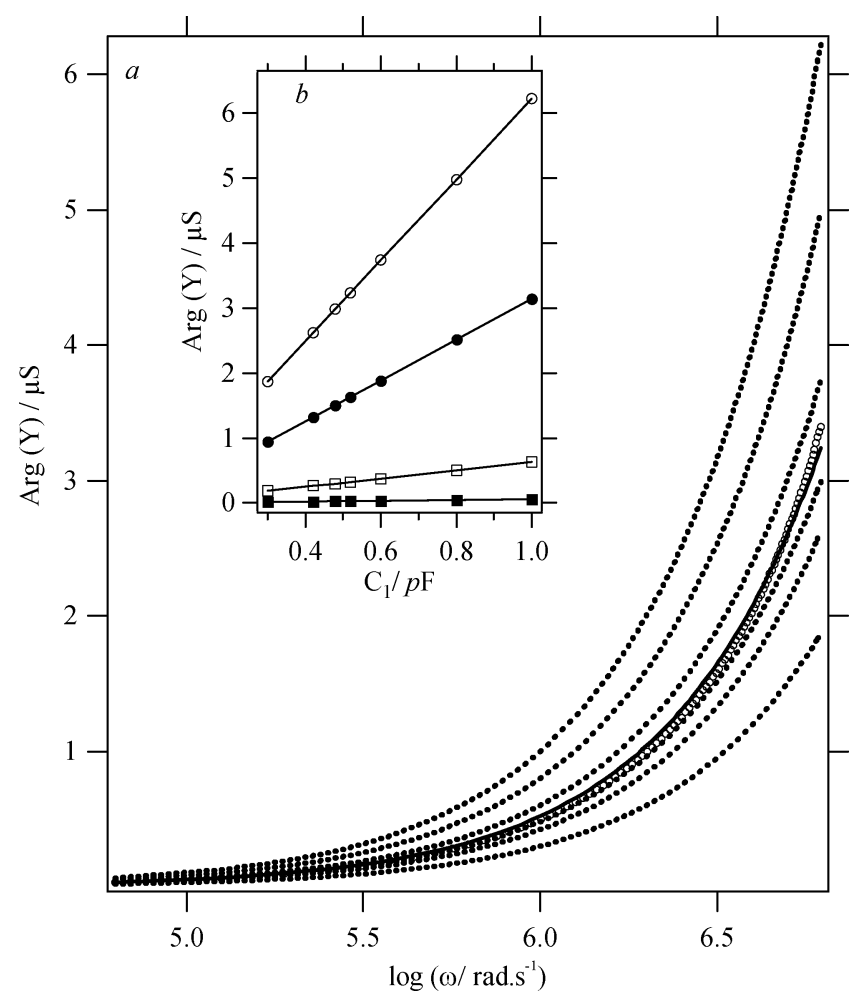

Figure 3. (a) Simulation of the admittance of the device with different capacitance. Dotted lines from bottom to top: $C_{1}=3 \times 10^{-13}, 4.2 \times 10^{-13}$, $4.8 \times 10^{-13}, 5.2 \times 10^{-13}, 6 \times 10^{-13}, 8 \times 10^{-13}$, and $1 \times 10^{-12} \mathrm{~F}$. Solid line: best simulation in the case of the adsorption of antibodies onto the surface of the modified channel. Hollowed spheres: experimental admittance obtained for the adsorption of antibodies. (b) Theoretical evolution of the admittance with respect to the capacitance of the PET/channel interface (hollowed spheres, $1 \mathrm{MHz}$; filled spheres, $500 \mathrm{kHz}$; hollowed squares, 100 $\mathrm{kHz}$; filled squares, $10 \mathrm{kHz}$ ).

displayed in Figure 3. It is interesting to notice the linear relation between the admittance of the device and the value of $C_{1}$. It should be noted that varying $R_{\mathrm{S}}$ in the simulation has a negligible effect on the imaginary response at high frequencies. This figure shows that the admittance is a direct measure of the charge state of the PET/solution interface. For example, during the process of adsorption of charged molecules onto the surface of the modified channel, the surface charge will be modified, and thus, the capacitance of the electrode/PET/channel will change. In the rest of this paper, it was decided to report only the impedance at a frequency of $1 \mathrm{MHz}$ so as to increase the sensitivity of measurement.

Due to the ability of tuning the capacitive character of the device by modifying the channel surface, the configuration can be employed for detecting biomolecules. To illustrate the application of the SCAT detection scheme, we present here an example of sensorgrams measured during the adsorption of $\mathrm{IgG}$ antibodies on the gold nanoparticle modified PET microchannel, as displayed in Figure 4. A set of experiments with variable IgG concentrations is performed, and the impedance at $1 \mathrm{MHz}$ is recorded versus the time. The release of the $\mathrm{IgG}$ is performed by rinsing the channel with the buffer solution. This process of release is allowed for low concentrations of $\mathrm{IgG}$, typically lower than $0.66 \mu \mathrm{M}$.

The axes are inverted to obtain sensorgrams of the same shapes as those routinely reported using SPR detection. For each IgG concentration, the adsorption of the IgG on gold nanoparticles induces the decrease of the impedance until a plateau

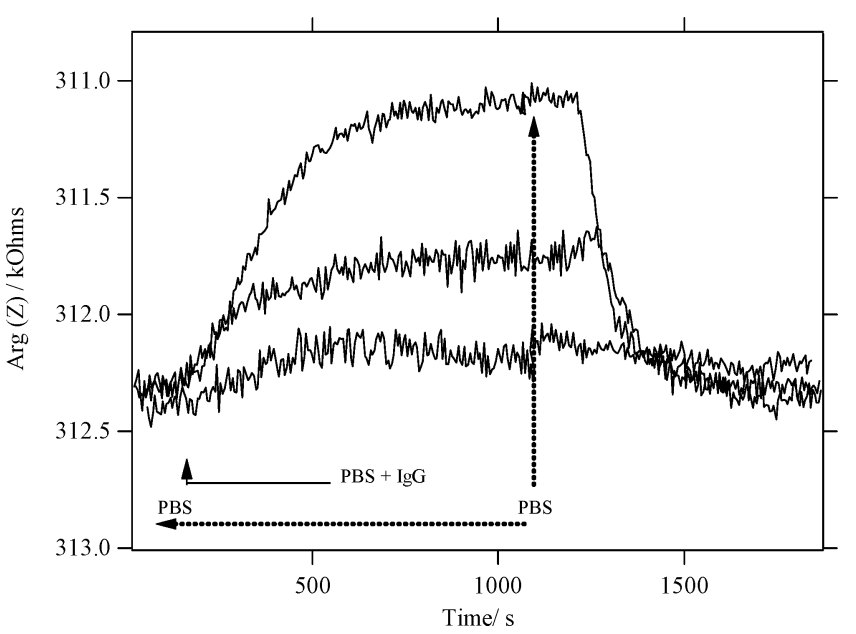

Figure 4. Sensorgrams showing the real time adsorption/desorption of antibodies. Before each experiment, a preliminary step consists of filling the microchannel with a buffer in order to obtain the baseline (buffer in). Then, in a second step, a sample of antibody is injected in a dynamic regime during $1200 \mathrm{~s}$; from bottom to top: $1.65 \times 10^{-9}, 6.6 \times 10^{-9}$, and $1.65 \times$ $10^{-8} \mathrm{M}$. The last stage is the desorption process by flowing the initial buffer solution. For all injections a typical flow of $0.1 \mu \mathrm{L} \mathrm{min}^{-1}$ is used. The flux is controlled by a dual syringe pump and a manual valve.

is reached, revealing that the equilibrium state is reached. In a second step, the microchannel is washed using a buffer solution, and an increase of the impedance is observed until the initial impedance value is reached. As IgGs are immobilized on the microchannel wall coated with gold nanoparticles, the impedance between the electrode surface and the solution flowing over it varies. This variation, caused by binding or desorption of IgG from the microchannel wall, is displayed in a sensorgram. The signal becomes constant once the interactions reach equilibrium and returns to the baseline level upon washing with buffer. This return to the baseline can only be obtained for diluted protein solution. Following the temporal evolution of the adsorption and desorption of $\mathrm{IgG}$ at the surface of the modified PET channel and using a Langmuir model, an equilibrium constant of $5.710^{7} \mathrm{M}^{-1}$ has been extracted. This value is in good agreement with previous constants reported for the adsorption of antigens on a gold surface. ${ }^{25}$

To quantify the adsorption process of IgG immunoglobulin molecules at the microchannel wall, further impedance measurements with respect to the concentration of $\mathrm{IgG}$ are performed. The evolution of the normalized impedance with respect to the concentration of $\mathrm{IgG}$ is displayed in Figure 5. The data corresponds to repetitive experiments. The impedance is normalized by $\operatorname{Arg}\left(Z_{\max }\right)$ that corresponds to the impedance reached when the surface is saturated with IgG immunoglobulins for a bulk concentration of $1 \mu \mathrm{M}$. With the assumption, in a first approximation, that the normalized impedance is related to the number of molecules adsorbed at the water/PET interface, the adsorption process follows the isotherm shown in Figure 5.

We have therefore demonstrated that the tunability of the surface charge upon chemical modification, such as adsorption phenomena, results in the decrease of the impedance that can be measured by the SCAT method described above. The experimental device provides detection with a good reproducibility and negligible signal background. Similar results have been observed for the detection of $\beta$-lactoglobulin, confirming

(25) Mullett, W. M.; Lai, E. P. C.; Yeung, J. M. Methods 2000, 22, 77-91. 


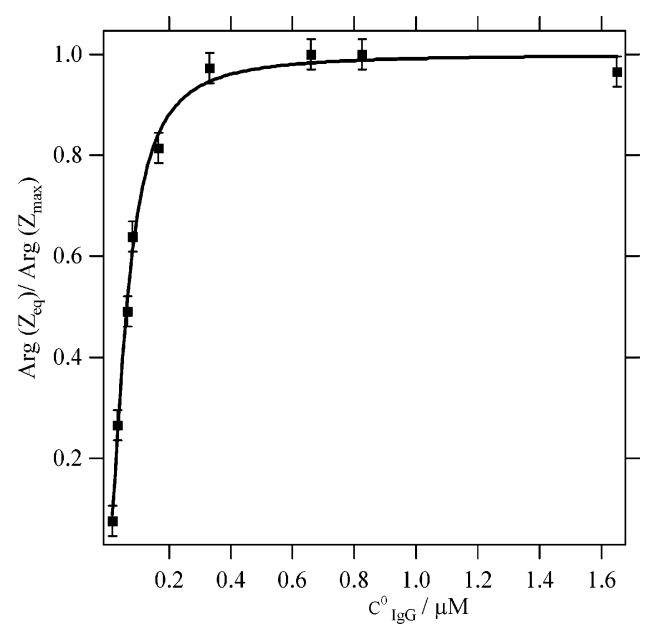

Figure 5. Evolution of the normalized impedance with respect to the $\operatorname{IgG}$ concentration.

that the device is not exclusively dedicated to a class of molecules and can be used as an online detection tool for any kind of macro- or biomolecules. The SCAT detection method probes the charge modification at the microchannel wall and, thus, the Gouy-Chapman layer in solution that corresponds to a probing depth of few nanometers. With the use of the SCAT technique, concentrations in the femtomolar range can be probed with a good reproducibility, and thus, the SCAT technique can be considered more sensitive than surface plasmon resonance. ${ }^{25}$

\section{Concluding Remarks}

To sum up, a microscale hybrid device with two planar microelectrodes in noncontact mode has been developed as a basic tool for the detection of the surface concentration of biomolecules with a good reproducibility. The device is designed to ensure the capacitive coupling between the electrodes and the solution to measure the capacitance of the solution/dielectric/ electrode system, contrary to noncontact conductivity detection in capillary electrophoresis where the resistive component is measured. ${ }^{26,27}$ The study shows that the response of the sensor is purely capacitive for a frequency close to $1 \mathrm{MHz}$, and any kind of surface modification of the channel induces a variation of the impedance. Supercapacitive admittance tomoscopy (SCAT) enables the study of binding mechanisms and the role of noncovalent forces involved in a complex formation in real time on any dielectric substrate with a modified surface such as nanoparticles-decorated PET. Thus, the SCAT approach is well suited for investigating the thermodynamics of an interaction. The flow sensor demonstrated above provides another tool for diagnostics and opens new ways for applications in the pharmaceutical industry. Additionally, the simplicity and the "multiuse" configuration in a restricted concentration range of the microchannel device can be considered as a crucial parameter for challenging other optical techniques in the biosensors domain.

Acknowledgment. The authors thank Valerie Devaud for her technical help and Solartron Switzerland for the loan of the dielectric interface 1296.

JA050602O

(26) Baltussen, E.; Guijt, R. M.; Van der Steen, G.; Laugere, F.; Baltussen, S.; Van Dedem, G. W. K. Electrophoresis 2002, 23 (17), 2888-2893.

(27) Abad-Villar, E. M.; Tanyanyiwa, J.; Fernandez-Abedul, M. T.; Costa-Garcia, A.; Hauser, P. C. Anal. Chem. 2004, 76, 1282-1288. 